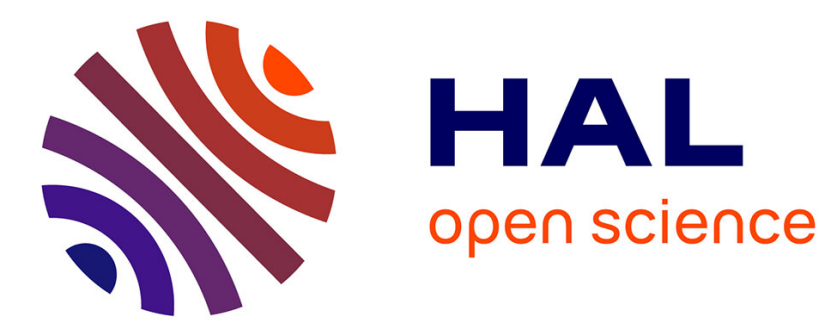

\title{
Evidence for a narrow anti-charmed baryon state
}

\author{
A. Aktas, V. Andreev, T. Anthonis, A. Asmone, A. Babaev, S. Backovic, J. \\ Bahr, P. Baranov, E. Barrelet, W. Bartel, et al.
}

\section{To cite this version:}

A. Aktas, V. Andreev, T. Anthonis, A. Asmone, A. Babaev, et al.. Evidence for a narrow anti-charmed baryon state. Physics Letters B, 2004, 588, pp.17-28. in2p3-00022141

\section{HAL Id: in2p3-00022141 https://hal.in2p3.fr/in2p3-00022141}

Submitted on $30 \mathrm{Jul} 2004$

HAL is a multi-disciplinary open access archive for the deposit and dissemination of scientific research documents, whether they are published or not. The documents may come from teaching and research institutions in France or abroad, or from public or private research centers.
L'archive ouverte pluridisciplinaire HAL, est destinée au dépôt et à la diffusion de documents scientifiques de niveau recherche, publiés ou non, émanant des établissements d'enseignement et de recherche français ou étrangers, des laboratoires publics ou privés. 
DESY 04-038

ISSN 0418-9833

March 2004

\title{
Evidence for a Narrow Anti-Charmed Baryon State
}

\author{
H1 Collaboration
}

\begin{abstract}
A narrow resonance in $D^{*-} p$ and $D^{*+} \bar{p}$ invariant mass combinations is observed in inelastic electron-proton collisions at centre-of-mass energies of $300 \mathrm{GeV}$ and $320 \mathrm{GeV}$ at HERA. The resonance has a mass of $3099 \pm 3$ (stat.) \pm 5 (syst.) $\mathrm{MeV}$ and a measured Gaussian width of $12 \pm 3$ (stat.) $\mathrm{MeV}$, compatible with the experimental resolution. The resonance is interpreted as an anti-charmed baryon with a minimal constituent quark composition of $u u d d \bar{c}$, together with the charge conjugate.
\end{abstract}

To be submitted to Phys. Lett. B 
A. Aktas $^{10}$, V. Andreev ${ }^{26}$, T. Anthonis ${ }^{4}$, A. Asmone ${ }^{33}$, A. Babaev ${ }^{25}$, S. Backovic ${ }^{37}$, J. Bähr ${ }^{37}$, P. Baranov ${ }^{26}$, E. Barrelet ${ }^{30}$, W. Bartel ${ }^{10}$, S. Baumgartner ${ }^{38}$, J. Becker ${ }^{39}$, M. Beckingham ${ }^{21}$, O. Behnke ${ }^{13}$, O. Behrendt ${ }^{7}$, A. Belousov ${ }^{26}$, Ch. Berger ${ }^{1}$, N. Berger ${ }^{38}$, T. Berndt ${ }^{14}$, J.C. Bizot ${ }^{28}$, J. Böhme ${ }^{10}$, M.-O. Boenig ${ }^{7}$, V. Boudry ${ }^{29}$, J. Bracinik ${ }^{27}$, V. Brisson ${ }^{28}$, H.-B. Bröker ${ }^{2}$, D.P. Brown ${ }^{10}$, D. Bruncko ${ }^{16}$, F.W. Büsser ${ }^{11}$, A. Bunyatyan ${ }^{12,36}$, G. Buschhorn ${ }^{27}$, L. Bystritskaya ${ }^{25}$, A.J. Campbell ${ }^{10}$, S. Caron ${ }^{1}$, F. Cassol-Brunner ${ }^{22}$, K. Cerny ${ }^{32}$, V. Chekeliann 27 , C. Collard ${ }^{4}$, J.G. Contreras ${ }^{23}$, Y.R. Coppens ${ }^{3}$, J.A. Coughlan ${ }^{5}$, B.E. Cox ${ }^{21}$, G. Cozzika ${ }^{9}$, J. Cvach ${ }^{31}$, J.B. Dainton ${ }^{18}$, W.D. Dau ${ }^{15}$, K. Daum ${ }^{35,41}$, B. Delcourt ${ }^{28}$, R. Demirchyan ${ }^{36}$, A. De Roeck ${ }^{10,44}$, K. Desch ${ }^{11}$, E.A. De Wolf ${ }^{4}$, C. Diaconu ${ }^{22}$, J. Dingfelder ${ }^{13}$, V. Dodonov ${ }^{12}$, A. Dubak ${ }^{27}$, C. Duprel ${ }^{2}$, G. Eckerlin ${ }^{10}$, V. Efremenko ${ }^{25}$, S. Egli ${ }^{34}$, R. Eichler ${ }^{34}$, F. Eisele ${ }^{13}$, M. Ellerbrock ${ }^{13}$, E. Elsen ${ }^{10}$, M. Erdmann ${ }^{10,42}$, W. Erdmann ${ }^{38}$, P.J.W. Faulkner ${ }^{3}$, L. Favart ${ }^{4}$, A. Fedotov ${ }^{25}$, R. Felst ${ }^{10}$, J. Ferencei ${ }^{10}$, M. Fleischer ${ }^{10}$, P. Fleischmann ${ }^{10}$, Y.H. Fleming ${ }^{10}$, G. Flucke ${ }^{10}$, G. Flügge ${ }^{2}$, A. Fomenko ${ }^{26}$, I. Foresti ${ }^{39}$, J. Formánek ${ }^{32}$, G. Franke ${ }^{10}$, G. Frising ${ }^{1}$, E. Gabathuler ${ }^{18}$, K. Gabathuler ${ }^{34}$, E. Garutti ${ }^{10}$, J. Garvey ${ }^{3}$, J. Gayler ${ }^{10}$, R. Gerhards ${ }^{10, \dagger}$, C. Gerlich ${ }^{13}$, S. Ghazaryan ${ }^{36}$,

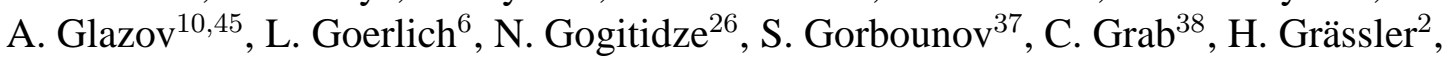
T. Greenshaw ${ }^{18}$, M. Gregori ${ }^{19}$, G. Grindhammer ${ }^{27}$, C. Gwilliam ${ }^{21}$, D. Haidt ${ }^{10}$, L. Hajduk ${ }^{6}$, J. Haller ${ }^{13}$, M. Hansson ${ }^{20}$, G. Heinzelmann ${ }^{11}$, R.C.W. Henderson ${ }^{17}$, H. Henschel ${ }^{37}$, O. Henshaw ${ }^{3}$, R. Heremans ${ }^{4}$, G. Herrera ${ }^{24}$, I. Herynek ${ }^{31}$, R.-D. Heuer ${ }^{11}$, M. Hildebrandt ${ }^{34}$, K.H. Hiller ${ }^{37}$, P. Höting ${ }^{2}$, D. Hoffmann ${ }^{22}$, R. Horisberger ${ }^{34}$, A. Hovhannisyan ${ }^{36}$, M. Ibbotson ${ }^{21}$, M. Ismail ${ }^{21}$, M. Jacquet ${ }^{28}$, L. Janauschek ${ }^{27}$, X. Janssen ${ }^{10}$, V. Jemanov ${ }^{11}$, L. Jönsson ${ }^{20}$, D.P. Johnson ${ }^{4}$, H. Jung ${ }^{20,10}$, D. Kant ${ }^{19}$, M. Kapichine ${ }^{8}$, M. Karlsson ${ }^{20}$, J. Katzy ${ }^{10}$, N. Keller ${ }^{39}$, J. Kennedy ${ }^{18}$, I.R. Kenyon ${ }^{3}$, C. Kiesling ${ }^{27}$, M. Klein ${ }^{37}$, C. Kleinwort ${ }^{10}$, T. Klimkovich ${ }^{10}$, T. Kluge ${ }^{1}$, G. Knies ${ }^{10}$, A. Knutsson ${ }^{20}$, B. Koblitz ${ }^{27}$, V. Korbel ${ }^{10}$, P. Kostka ${ }^{37}$, R. Koutouev ${ }^{12}$, A. Kropivnitskaya ${ }^{25}$, J. Kroseberg ${ }^{39}$, J. Kückens ${ }^{10}$, T. Kuhr ${ }^{10}$, M.P.J. Landon ${ }^{19}$, W. Lange ${ }^{37}$, T. Laštovička ${ }^{37,32}$, P. Laycock ${ }^{18}$, A. Lebedev ${ }^{26}$, B. Leißner ${ }^{1}$, R. Lemrani ${ }^{10}$, V. Lendermann ${ }^{14}$, S. Levonian ${ }^{10}$, L. Lindfeld ${ }^{39}$, K. Lipka ${ }^{37}$, B. List ${ }^{38}$, E. Lobodzinska ${ }^{37,6}$,

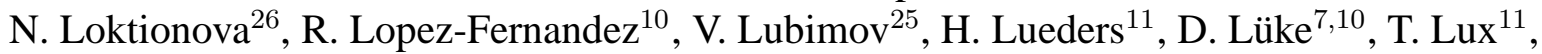
L. Lytkin ${ }^{12}$, A. Makankine ${ }^{8}$, N. Malden ${ }^{21}$, E. Malinovski ${ }^{26}$, S. Mangano ${ }^{38}$, P. Marage ${ }^{4}$, J. Marks ${ }^{13}$, R. Marshall ${ }^{21}$, M. Martisikova ${ }^{10}$, H.-U. Martyn ${ }^{1}$, S.J. Maxfield ${ }^{18}$, D. Meer ${ }^{38}$, A. Mehta ${ }^{18}$, K. Meier ${ }^{14}$, A.B. Meyer ${ }^{11}$, H. Meyer ${ }^{35}$, J. Meyer ${ }^{10}$, S. Michine ${ }^{26}$, S. Mikocki ${ }^{6}$, I. Milcewicz ${ }^{6}$, D. Milstead ${ }^{18}$, A. Mohamed ${ }^{18}$, F. Moreau ${ }^{29}$, A. Morozov ${ }^{8}$, I. Morozov, J.V. Morris ${ }^{5}$, M.U. Mozer ${ }^{13}$, K. Müller ${ }^{39}$, P. Murín ${ }^{16,43}$, V. Nagovizin ${ }^{25}$, B. Naroska ${ }^{11}$, J. Naumann ${ }^{7}$, Th. Naumann ${ }^{37}$, P.R. Newman ${ }^{3}$, C. Niebuhr ${ }^{10}$, A. Nikiforov ${ }^{27}$, D. Nikitin ${ }^{8}$, G. Nowak ${ }^{6}$, M. Nozicka ${ }^{32}$, R. Oganezov ${ }^{36}$, B. Olivier ${ }^{10}$, J.E. Olsson ${ }^{10}$, G.Ossoskov $^{8}$, D. Ozerov ${ }^{25}$, C. Pascaud ${ }^{28}$, G.D. Patel ${ }^{18}$, M. Peez ${ }^{29}$, E. Perez ${ }^{9}$, A. Perieanu ${ }^{10}$, A. Petrukhin ${ }^{25}$, D. Pitzl ${ }^{10}$, R. Plačakytè ${ }^{27}$, R. Pöschl ${ }^{10}$, B. Portheault ${ }^{28}$, B. Povh ${ }^{12}$, N. Raicevic ${ }^{37}$, Z. Ratiani ${ }^{10}$, P. Reimer ${ }^{31}$, B. Reisert ${ }^{27}$, A. Rimmer ${ }^{18}$, C. Risler ${ }^{27}$, E. Rizvi ${ }^{3}$, P. Robmann ${ }^{39}$, B. Roland ${ }^{4}$, R. Roosen ${ }^{4}$, A. Rostovtsev ${ }^{25}$, Z. Rurikova ${ }^{27}$, S. Rusakov ${ }^{26}$, K. Rybicki ${ }^{6, \dagger}$, D.P.C. Sankey ${ }^{5}$, E. Sauvan ${ }^{22}$, S. Schätzel ${ }^{13}$, J. Scheins ${ }^{10}$, F.-P. Schilling ${ }^{10}$, P. Schleper ${ }^{10}$, S. Schmidt ${ }^{27}$, S. Schmitt ${ }^{39}$, M. Schneider ${ }^{22}$, L. Schoeffel ${ }^{9}$, A. Schöning ${ }^{38}$, V. Schröder ${ }^{10}$, H.-C. Schultz-Coulon ${ }^{14}$, C. Schwanenberger ${ }^{10}$, K. Sedlák ${ }^{31}$, F. Sefkow ${ }^{10}$, I. Sheviakov ${ }^{26}$, L.N. Shtarkov ${ }^{26}$, Y. Sirois ${ }^{29}$, T. Sloan ${ }^{17}$, P. Smirnov ${ }^{26}$, Y. Soloviev ${ }^{26}$, D. South ${ }^{10}$, V. Spaskov ${ }^{8}$, A. Specka ${ }^{29}$, H. Spitzer ${ }^{11}$, R. Stamen ${ }^{10}$, B. Stella ${ }^{33}$, J. Stiewe ${ }^{14}$, I. Strauch ${ }^{10}$, U. Straumann ${ }^{39}$, V. Tchoulakov ${ }^{8}$, G. Thompson ${ }^{19}$, P.D. Thompson ${ }^{3}$, F. Tomasz ${ }^{14}$, D. Traynor ${ }^{19}$, P. Truöl ${ }^{39}$, G. Tsipolitis ${ }^{10,40}$, I. Tsurin ${ }^{37}$, J. Turnau ${ }^{6}$, E. Tzamariudaki ${ }^{27}$, A. $\operatorname{Uraev}^{25}$, M. Urban ${ }^{39}$, 
A. Usik ${ }^{26}$, D. Utkin ${ }^{25}$, S. Valkár ${ }^{32}$, A. Valkárováa ${ }^{32}$, C. Vallée ${ }^{22}$, P. Van Mechelen ${ }^{4}$, N. Van Remortel $^{4}$, A. Vargas Trevino ${ }^{7}$, Y. Vazdik ${ }^{26}$, C. Veelken ${ }^{18}$, A. Vest ${ }^{1}$, S. Vinokurova ${ }^{10}$, V. Volchinski ${ }^{36}$, K. Wacker ${ }^{7}$, J. Wagner ${ }^{10}$, G. Weber ${ }^{11}$, R. Weber ${ }^{38}$, D. Wegener ${ }^{7}$, C. Werner ${ }^{13}$, N. Werner ${ }^{39}$, M. Wessels ${ }^{1}$, B. Wessling ${ }^{11}$, G.-G. Winter ${ }^{10}$, Ch. Wissing ${ }^{7}$, E.-E. Woehrling ${ }^{3}$, R. Wolf ${ }^{13}$, E. Wünsch ${ }^{10}$, S. Xella ${ }^{39}$, W. Yan ${ }^{10}$, V. Yeganov ${ }^{36}$, J. Žáček ${ }^{32}$, J. Zálešák ${ }^{31}$, Z. Zhang ${ }^{28}$, A. Zhokin ${ }^{25}$, H. Zohrabyan ${ }^{36}$, and F. Zomer ${ }^{28}$

${ }^{1}$ I. Physikalisches Institut der RWTH, Aachen, Germany ${ }^{a}$

${ }^{2}$ III. Physikalisches Institut der RWTH, Aachen, Germany ${ }^{a}$

${ }^{3}$ School of Physics and Astronomy, University of Birmingham, Birmingham, $U K^{b}$

${ }^{4}$ Inter-University Institute for High Energies ULB-VUB, Brussels; Universiteit Antwerpen (UIA), Antwerpen; Belgium ${ }^{c}$

${ }^{5}$ Rutherford Appleton Laboratory, Chilton, Didcot, $U K^{b}$

${ }^{6}$ Institute for Nuclear Physics, Cracow, Poland ${ }^{d}$

${ }^{7}$ Institut für Physik, Universität Dortmund, Dortmund, Germany ${ }^{a}$

${ }^{8}$ Joint Institute for Nuclear Research, Dubna, Russia

${ }^{9}$ CEA, DSM/DAPNIA, CE-Saclay, Gif-sur-Yvette, France

${ }^{10}$ DESY, Hamburg, Germany

${ }^{11}$ Institut für Experimentalphysik, Universität Hamburg, Hamburg, Germany ${ }^{a}$

${ }^{12}$ Max-Planck-Institut für Kernphysik, Heidelberg, Germany

${ }^{13}$ Physikalisches Institut, Universität Heidelberg, Heidelberg, Germany ${ }^{a}$

${ }^{14}$ Kirchhoff-Institut für Physik, Universität Heidelberg, Heidelberg, Germany ${ }^{a}$

${ }^{15}$ Institut für experimentelle und Angewandte Physik, Universität Kiel, Kiel, Germany

${ }^{16}$ Institute of Experimental Physics, Slovak Academy of Sciences, Košice, Slovak Republic e,f

${ }^{17}$ Department of Physics, University of Lancaster, Lancaster, $U K^{b}$

${ }^{18}$ Department of Physics, University of Liverpool, Liverpool, $U K^{b}$

${ }^{19}$ Queen Mary and Westfield College, London, $U K^{b}$

${ }^{20}$ Physics Department, University of Lund, Lund, Sweden ${ }^{g}$

${ }^{21}$ Physics Department, University of Manchester, Manchester, $U K^{b}$

${ }^{22}$ CPPM, CNRS/IN2P3 - Univ Mediterranee, Marseille - France

${ }^{23}$ Departamento de Fisica Aplicada, CINVESTAV, Mérida, Yucatán, México ${ }^{k}$

${ }^{24}$ Departamento de Fisica, CINVESTAV, México ${ }^{k}$

${ }^{25}$ Institute for Theoretical and Experimental Physics, Moscow, Russial

${ }^{26}$ Lebedev Physical Institute, Moscow, Russia ${ }^{e}$

${ }^{27}$ Max-Planck-Institut für Physik, München, Germany

${ }^{28}$ LAL, Université de Paris-Sud, IN2P3-CNRS, Orsay, France

${ }^{29}$ LLR, Ecole Polytechnique, IN2P3-CNRS, Palaiseau, France

${ }^{30}$ LPNHE, Universités Paris VI and VII, IN2P3-CNRS, Paris, France

${ }^{31}$ Institute of Physics, Academy of Sciences of the Czech Republic, Praha, Czech Republic ${ }^{e, i}$

${ }^{32}$ Faculty of Mathematics and Physics, Charles University, Praha, Czech Republic ${ }^{e, i}$

${ }^{33}$ Dipartimento di Fisica Università di Roma Tre and INFN Roma 3, Roma, Italy

${ }^{34}$ Paul Scherrer Institut, Villigen, Switzerland

${ }^{35}$ Fachbereich Physik, Bergische Universität Gesamthochschule Wuppertal, Wuppertal, Germany

${ }^{36}$ Yerevan Physics Institute, Yerevan, Armenia

${ }^{37}$ DESY, Zeuthen, Germany 
${ }^{38}$ Institut für Teilchenphysik, ETH, Zürich, Switzerland ${ }^{j}$

${ }^{39}$ Physik-Institut der Universität Zürich, Zürich, Switzerland ${ }^{j}$

${ }^{40}$ Also at Physics Department, National Technical University, Zografou Campus, GR-15773 Athens, Greece

${ }^{41}$ Also at Rechenzentrum, Bergische Universität Gesamthochschule Wuppertal, Germany

${ }^{42}$ Also at Institut für Experimentelle Kernphysik, Universität Karlsruhe, Karlsruhe, Germany

${ }^{43}$ Also at University of P.J. Šafárik, Košice, Slovak Republic

${ }^{44}$ Also at CERN, Geneva, Switzerland

${ }^{45}$ Also at University of Chicago, Enrico Fermi Institute, USA

$\dagger^{\dagger}$ Deceased

${ }^{a}$ Supported by the Bundesministerium für Bildung und Forschung, FRG, under contract numbers 05 H1 1GUA /1, 05 H1 1PAA /1, 05 H1 1PAB /9, 05 H1 1PEA /6, 05 H1 1VHA /7 and $05 \mathrm{H} 1 \mathrm{IVHB} / 5$

${ }^{b}$ Supported by the UK Particle Physics and Astronomy Research Council, and formerly by the UK Science and Engineering Research Council

${ }^{c}$ Supported by FNRS-FWO-Vlaanderen, IISN-IIKW and IWT

${ }^{d}$ Partially Supported by the Polish State Committee for Scientific Research, SPUB/DESY/P003/DZ 118/2003/2005

e Supported by the Deutsche Forschungsgemeinschaft

${ }^{f}$ Supported by VEGA SR grant no. 2/1169/2001

${ }^{g}$ Supported by the Swedish Natural Science Research Council

${ }^{i}$ Supported by the Ministry of Education of the Czech Republic under the projects INGO-LA116/2000 and LNOOA006, by GAUK grant no 173/2000

${ }^{j}$ Supported by the Swiss National Science Foundation

${ }^{k}$ Supported by CONACYT, México, grant 400073-F

${ }^{l}$ Partially Supported by Russian Foundation for Basic Research, grant no. 00-15-96584

Dedicated to the memory of our dear friend and colleague, Ralf Gerhards. 


\section{Introduction}

Several experiments have recently reported the observation of a narrow resonance with mass in the region of $1540 \mathrm{MeV}$, decaying to $K^{+} n$ or $K_{s}^{0} p$ [1]. This state has both baryon number and strangeness of +1 , such that its minimal composition in the constituent quark model is $u u d d \bar{s}$. It has thus been interpreted as a pentaquark [2,3], the $\theta^{+}$. There is also evidence for two related states with strangeness of -2 [4]. Various models have been put forward to explain the nature of these states and the structure of the multiplet that contains them $[3,5,6]$. The possibility of pentaquark states in the charm sector has also been discussed [7], with renewed theoretical interest in calculating their expected properties $[5,8]$ following the observation of strange pentaquarks.

The electron-proton collider, HERA, is a copious producer of both charm and anti-charm quarks, the dominant production mechanism being boson-gluon fusion, $\gamma^{(\star)} g \rightarrow c \bar{c}$. The spectroscopy of charmed hadrons can be studied using the final states to which the quarks and anti-quarks hadronise. This paper reports the first evidence for a baryon with exotic quantum numbers in the charm sector, using deep inelastic scattering (DIS) data taken with the H1 detector. A resonance is observed when combining $D^{*-} \rightarrow \bar{D}^{0} \pi_{s}^{-} \rightarrow K^{+} \pi^{-} \pi_{s}^{-}$candidates with proton candidates and when combining $D^{*+} \rightarrow D^{0} \pi_{s}^{+} \rightarrow K^{-} \pi^{+} \pi_{s}^{+}$candidates with antiproton candidates. ${ }^{1}$ The resonance is also observed in an independent photoproduction data sample.

\section{Experimental Procedure}

\subsection{H1 Apparatus}

The tracks from charged particles used in this analysis are reconstructed in the $\mathrm{H} 1$ central tracker, whose main components are two cylindrical drift chambers, the inner and outer central jet chambers (CJCs), covering the polar angle region ${ }^{2} 20^{\circ}<\theta<160^{\circ}$. The inner and outer CJCs are mounted concentrically around the beam-line, have 24 and 32 sense wires, respectively, and cover radii between $20 \mathrm{~cm}$ and $84 \mathrm{~cm}$. The information from the CJC sense wires is digitised using $100 \mathrm{MHz}$ FADCs, providing simultaneous charge and timing measurements. The CJCs lie within a homogeneous magnetic field of $1.15 \mathrm{~T}$, which allows measurements of the transverse momenta of charged particles. Two additional drift chambers complement the CJCs by precisely measuring the $z$ coordinates of track segments and hence assist in the determination of the particle's polar angle. The Central Silicon Tracker, consisting of two layers at radii of $6 \mathrm{~cm}$ and $10 \mathrm{~cm}$, is also used to improve the charged particle track and event vertex reconstruction. The transverse momentum resolution of the central tracker is $\sigma\left(p_{\mathrm{T}}\right) / p_{\mathrm{T}} \simeq 0.005 p_{\mathrm{T}}[\mathrm{GeV}] \oplus 0.015$. The charge misidentification probability is negligible

\footnotetext{
${ }^{1}$ In the remainder of this paper, particle charges are not generally given. Both charge conjugate configurations are always implied, unless explicitly stated otherwise. The notation $\pi_{s}$ is used to distinguish the low momentum pion released in the $D^{*}$ decay from that from the $D^{0}$ decay.

${ }^{2}$ The H1 experiment uses a coordinate system in which the positive $z$-axis is defined by the direction of the outgoing proton beam. The polar angle $\theta$ of a particle is defined relative to this axis and is related to the pseudorapidity $\eta$ by $\eta=-\ln \tan \theta / 2$.
} 
for particles originating from the primary vertex which have transverse momenta in the range relevant to this analysis.

The specific ionisation energy loss of charged particles is derived from the mean of the inverse square-root of the charge collected by all CJC sense wires with a signal above threshold. The resolution is $\sigma(\mathrm{d} E / \mathrm{d} x) /(\mathrm{d} E / \mathrm{d} x) \simeq 8 \%$ on average for minimum ionising particles [9].

A lead/scintillating-fibre spaghetti calorimeter (SpaCal) is located in the direction of the outgoing electron beam. It contains both electromagnetic and hadronic sections and is used to detect the scattered electron in DIS events. The global properties of the hadronic final state are reconstructed using an algorithm which takes information from the central tracker, the SpaCal, and also from a Liquid Argon calorimeter, which surrounds the central tracker. The DIS events studied in this paper are triggered on the basis of a scattered electron in the SpaCal, complemented by the signals in the CJCs and multi-wire proportional chambers in the central tracker. Further details of the $\mathrm{H} 1$ detector can be found in [10].

\subsection{The DIS Data Sample}

The analysis is carried out using data taken in the years 1996-2000, when HERA collided electrons $^{3}$ of energy $27.6 \mathrm{GeV}$ with protons at $820 \mathrm{GeV}$ (1996-1997) and $920 \mathrm{GeV}$ (1998-2000). The integrated luminosity of the sample is $75 \mathrm{pb}^{-1}$.

The scattered electron energy, measured in the SpaCal, is required to be above $8 \mathrm{GeV}$, and the virtuality of the exchanged photon ${ }^{4}$ is required to lie in the range $1<Q^{2}<100 \mathrm{GeV}^{2}$, as reconstructed from the energy and polar angle of the electron. To ensure that the hadronic final state lies in the central region of the detector, the inelasticity of the event is required to satisfy $0.05<y<0.7$, calculated using the scattered electron kinematics. The $z$ coordinate of the event vertex, reconstructed using the central tracker, is required to lie within $35 \mathrm{~cm}$ of the mean position for $e p$ interactions. The difference between the total energy $E$ and the longitudinal component of the total momentum $p_{z}$, calculated from the electron and the hadronic final state, is restricted to $E-p_{z}>35 \mathrm{GeV}$. This requirement suppresses photoproduction background, where a hadron fakes the electron signature.

\subsection{Selection of $D^{*}$ Meson and Proton Candidates}

The decay channel $D^{*} \rightarrow D^{0} \pi_{s} \rightarrow K \pi \pi_{s}$ is used to reconstruct $D^{*}$ mesons. The charged particle selection criteria, $\mathrm{d} E / \mathrm{d} x$ requirements and transverse momentum cuts on the decay products are very similar to those used in previous $\mathrm{H} 1$ analyses [11]. Unlike-charge particle combinations are made to form $K^{\mp} \pi^{ \pm}$pairs, where the particles are required to be consistent with kaons and pions according to their $\mathrm{d} E / \mathrm{d} x$ measurements. Those combinations that give rise to an invariant mass within $60 \mathrm{MeV}$ of the nominal $D^{0}$ mass of $1864.5 \mathrm{MeV}$ [12] are

\footnotetext{
${ }^{3}$ The analysis uses data from periods when the beam lepton was either a positron ( $88 \%$ of the total) or an electron ( $12 \%$ of the total).

${ }^{4}$ The inclusive DIS kinematic variables are defined as $Q^{2}=-q^{2}, y=q \cdot p / k \cdot p$ and $x=-q^{2} / 2 q \cdot p$, where $q, k$ and $p$ are the 4-vectors of the exchanged photon, the incident electron and the incident proton, respectively.
} 
combined with further pion candidates $\left(\pi_{s}\right)$ with opposite charge to the kaon. To obtain good experimental resolution and background rejection in the $D^{*}$ reconstruction, the standard mass difference technique [13] is used, based on the variable

$$
\Delta M_{D^{*}}=m\left(K \pi \pi_{s}\right)-m(K \pi) .
$$

The sample is restricted to a region where the background to the $D^{*}$ signal is relatively small by requiring that the $D^{*}$ candidates have transverse momentum $p_{\mathrm{T}}\left(D^{*}\right)>1.5 \mathrm{GeV}$, pseudorapidity $-1.5<\eta\left(D^{*}\right)<1$ and production elasticity $z\left(D^{*}\right)=\left(E-p_{z}\right)_{D^{*}} / 2 y E_{e}>0.2$, where $E_{e}$ is the electron beam energy.

The resulting $\Delta M_{D^{*}}$ distribution is shown in figure 1a. Here and elsewhere in this paper, the error bars shown represent the square-root of the numbers of entries in each bin. A prominent signal on a smooth background is observed around the expected $D^{*}-D^{0}$ mass difference.

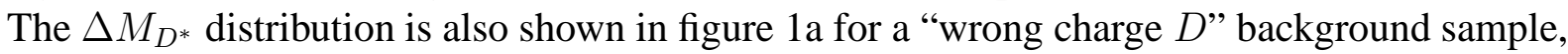
which is formed by $K^{ \pm} \pi^{ \pm}$combinations in the accepted $D^{0}$ mass range. The "wrong charge $D$ " distribution gives a good description of the correct-charge $D^{0}$ combinations away from the $D^{*}$ peak. Candidate $D^{*}$ mesons for which $\Delta M_{D^{*}}$ lies within $\pm 2.5 \mathrm{MeV}$ of the nominal mass difference $m\left(D^{*}\right)-m\left(D^{0}\right)=145.4 \mathrm{MeV}$ [12] are selected for further analysis.

Proton candidates are selected using requirements on the particle $\mathrm{d} E / \mathrm{d} x$ measurements. Figure $1 \mathrm{~b}$ shows the $\mathrm{d} E / \mathrm{d} x$ values, plotted against momentum, for a sample of particles which yield a mass $M\left(D^{*} p\right)<3.9 \mathrm{GeV}$ (see equation 2 ) when combined with $K \pi \pi_{s}$ candidates falling in the accepted $\Delta M_{D^{*}}$ region. The likelihoods that a particle is a pion, kaon or proton are obtained from the proximity of the measured $\mathrm{d} E / \mathrm{d} x$ to the most probable values for each particle type at the reconstructed momentum. The most probable $\mathrm{d} E / \mathrm{d} x$ values are derived from phenomenological parameterisations [9], shown in figure 1b, which are based on the Bethe-Bloch formula. The normalised proton likelihood $L_{p}$ is defined to be the ratio of the proton likelihood to the sum of the pion, kaon and proton likelihoods. For momenta $p(p)<2 \mathrm{GeV}$, a requirement $L_{p}>0.3$ is applied, which selects protons where they are clearly identified at low momentum and suppresses contributions close to the crossing points of the proton, pion and kaon parameterisations. For $p(p)>2 \mathrm{GeV}$, the requirement is loosened to $L_{p}>0.1$, which suppresses background from particles with large $\mathrm{d} E / \mathrm{d} x$ such as electrons. The main selection criteria are summarised in table 1.

\section{Analysis of $D^{*} p$ Combinations}

\section{1 $\quad D^{*} p$ Invariant Mass Distributions}

The $D^{*}$ and proton candidates are combined to form the mass difference $m\left(K \pi \pi_{s} p\right)-m\left(K \pi \pi_{s}\right)$, to which the $D^{*}$ mass of $2010.0 \mathrm{MeV}$ [12] is added to obtain the mass of the $D^{*} p$ combination. The distributions in

$$
M\left(D^{*} p\right)=m\left(K \pi \pi_{s} p\right)-m\left(K \pi \pi_{s}\right)+m\left(D^{*}\right)
$$

are shown in figure 2a for "opposite-charge" $D^{*} p$ combinations $\left(K^{-} \pi^{+} \pi_{s}^{+} \bar{p}\right.$ and $\left.K^{+} \pi^{-} \pi_{s}^{-} p\right)$ and in figure $2 \mathrm{~b}$ for "same-charge" $D^{*} p$ combinations $\left(K^{-} \pi^{+} \pi_{s}^{+} p\right.$ and $\left.K^{+} \pi^{-} \pi_{s}^{-} \bar{p}\right)$. A clear 

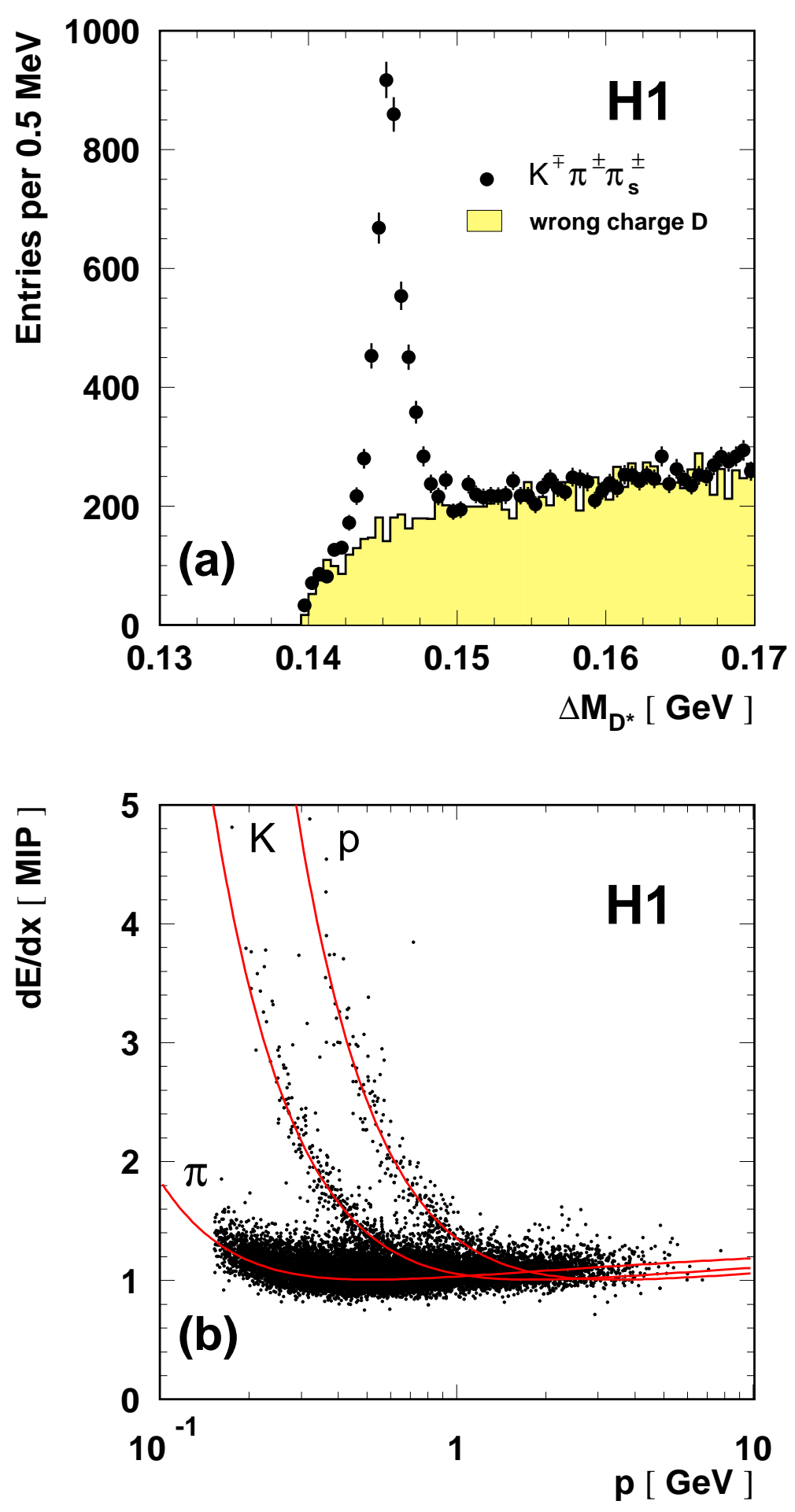

Figure 1: (a) $\Delta M_{D^{*}}$ distribution for $K^{\mp} \pi^{ \pm} \pi_{s}^{ \pm}$combinations as described in the text. For comparison, the distribution from "wrong charge $D$ " combinations, where the $K$ and $\pi$ yielding the $D^{0}$ mass have the same charge, is also shown. (b) Specific ionisation energy loss relative to that of a minimally ionising particle, plotted against momentum, for the sample described in the text. The curves indicate parameterisations of the most probable responses of the CJCs for pions, kaons and protons. 


\begin{tabular}{|l|l|}
\hline $\boldsymbol{D}^{\mathbf{0}}$ & $p_{\mathrm{T}}(K)>500 \mathrm{MeV}$ \\
& $p_{\mathrm{T}}(\pi)>250 \mathrm{MeV}$ \\
& $p_{\mathrm{T}}(K)+p_{\mathrm{T}}(\pi)>2 \mathrm{GeV}$ \\
& $\left|m(K \pi)-m\left(D^{0}\right)\right|<60 \mathrm{MeV}$ \\
\hline $\boldsymbol{D}^{*}$ & $p_{\mathrm{T}}\left(\pi_{s}\right)>120 \mathrm{MeV}$ \\
& $\left|\Delta M_{D^{*}}-m\left(D^{*}\right)+m\left(D^{0}\right)\right|<2.5 \mathrm{MeV}$ \\
& $p_{\mathrm{T}}\left(D^{*}\right)>1.5 \mathrm{GeV}$ \\
& $-1.5<\eta\left(D^{*}\right)<1$ \\
& $z\left(D^{*}\right)>0.2$ \\
\hline $\boldsymbol{p}$ & $p_{\mathrm{T}}(p)>120 \mathrm{MeV}$ \\
& $L_{p}>0.3$ for $p(p)<2 \mathrm{GeV}$ \\
& $L_{p}>0.1$ for $p(p)>2 \mathrm{GeV}$ \\
\hline
\end{tabular}

Table 1: Summary of the kinematic and proton energy loss selection criteria applied to define the $D^{*}$ and proton candidates.

and narrow peak is observed for the opposite-charge combinations at $M\left(D^{*} p\right) \simeq 3100 \mathrm{MeV}$. Approximately half of the events in this signal arise from each of the $D^{*-} p$ and $D^{*+} \bar{p}$ combinations (see section 4). The distribution for the same-charge combinations shows a small enhancement in the $M\left(D^{*} p\right)$ region in which the opposite-charge signal is observed.

The background distributions for the $D^{*} p$ combinations are modelled by the sum of two contributions, which are shown in figure 2. Background from random combinations not involving charm is modelled using the "wrong charge $D$ " combinations, as described in section 2.3, combined with proton candidates as for the correct-charge $D^{0}$ sample. Combinatorial background from $D^{*}$ mesons with real or misidentified protons is modelled using simulated events from the RAPGAP [14] Monte Carlo model applied to $D^{*}$ production in DIS, including string fragmentation and decays from JETSET $[15,16]$. The RAPGAP model gives a good description of the shapes of the inclusive $D^{*}$ distributions. This contribution (" $D^{*} \mathrm{MC}$ " in figure 2 ) is normalised according to the $D^{*}$ yield in the data (figure 1a).

No significant structures are observed in either component of this background model. The model gives a reasonable description of the shape and normalisation of the $M\left(D^{*} p\right)$ distribution away from the signal region for the opposite-charge combinations. The $M\left(D^{*} p\right)$ distribution from the same-charge combinations is also well described in shape, though the model prediction lies approximately $15 \%$ above the data.

Alternative models have been studied for the background distribution for the oppositecharge $D^{*} p$ combinations. Similar distributions to those shown in figure 2 are obtained when a DJANGO [17] simulation of inclusive DIS is used to replace both model components. The same is true when the RAPGAP model of the $D^{*}$-related background is replaced by simulations with modified parton shower dynamics (CASCADE [18]) or fragmentation (HERWIG [19]). In all cases, no resonant structures are observed in the simulated $M\left(D^{*} p\right)$ distributions. Possible contributions from beauty decays have been considered using a further RAPGAP Monte Carlo simulation. After normalising to the luminosity of the data, the resulting contribution is negligible. 

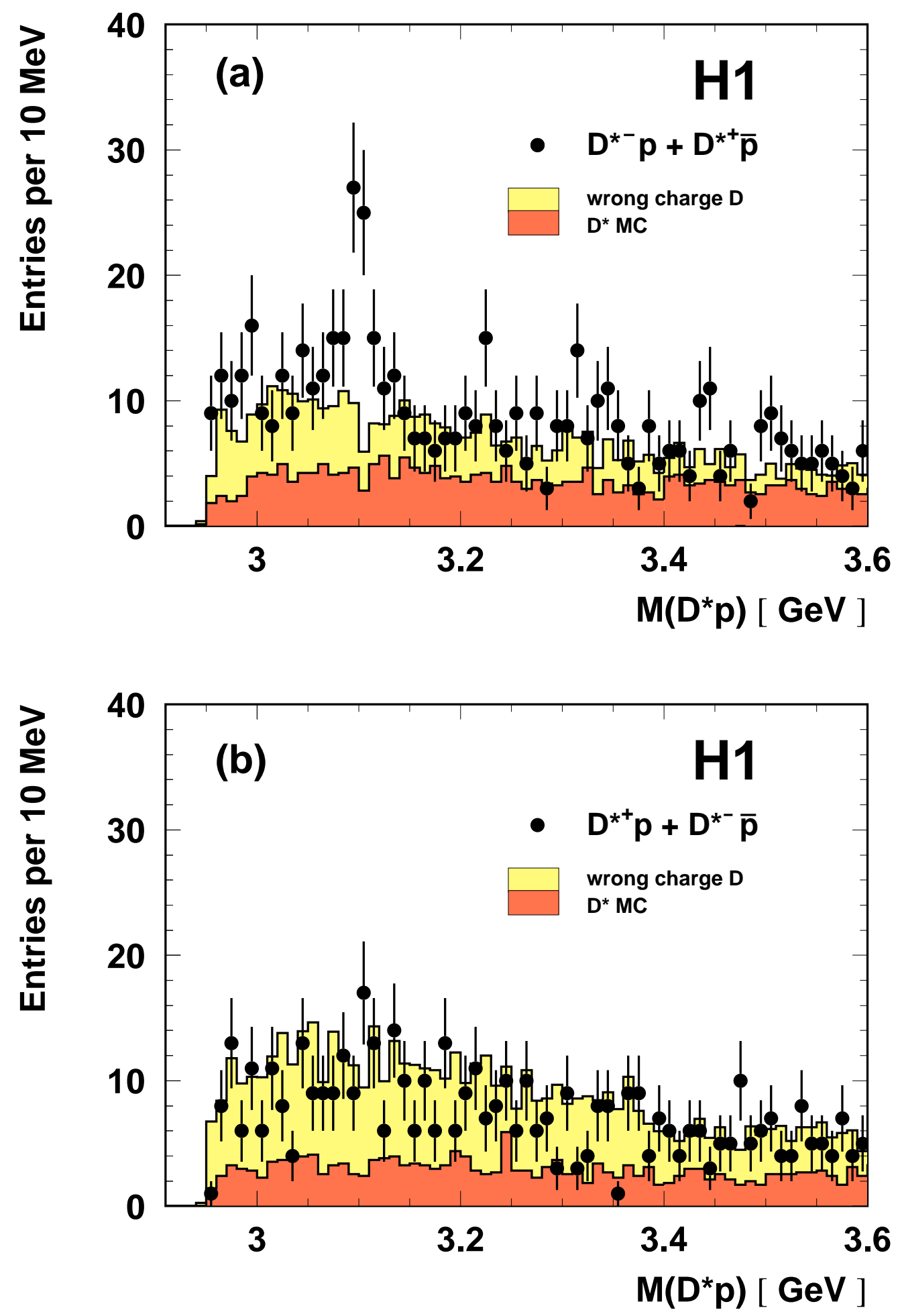

Figure 2: Distributions in $M\left(D^{*} p\right)$ for (a) opposite-charge and (b) same-charge $D^{*} p$ combinations. The data are compared with a two-component background model in which "wrong charge $D$ " $K^{ \pm} \pi^{ \pm}$combinations are used to describe non-charm related background and the " $D^{*} \mathrm{MC}$ " simulation describes background involving real $D^{*}$ mesons. 
The events giving contributions in the peak region of the opposite-charge $M\left(D^{*} p\right)$ distribution have been visually scanned and no anomalies are observed in the events or the candidate tracks. All entries within $\pm 24 \mathrm{MeV}$ of the peak arise from different events, with one exception where the same $\pi, \pi_{s}$ and proton candidates contribute with two different $K$ candidates. For the full $M\left(D^{*} p\right)$ range shown in figure $2 \mathrm{a}$, there are an average of 1.12 entries per event. The signal is present in each case when the data are divided into two sub-samples of similar size, discriminated in variables such as $x$ or $Q^{2}$, the pseudorapidity or transverse momentum of the $D^{*} p$ composite, or the data taking period. The peak also remains clearly visible for reasonable variations in the binning or selection criteria. In all cases, the observed mass and width of the peak are stable to within a few $\mathrm{MeV}$.

\subsection{Particle Identification Tests}

The $D^{*}$ and proton content of the signal in the mass distribution from opposite-charge $D^{*} p$ combinations has been investigated in complementary studies. The $D^{*}$ content is tested by forming the $M\left(D^{*} p\right)$ distribution (equation 2) with the full proton selection, but with no requirement on $\Delta M_{D^{*}}$. The $\Delta M_{D^{*}}$ distribution (equation 1) is shown in figure 3 for events in a $\pm 15 \mathrm{MeV}$ window around the $D^{*} p$ signal $\left(3085<M\left(D^{*} p\right)<3115 \mathrm{MeV}\right)$. For comparison, a similar distribution is shown, taken from side bands with $2990<M\left(D^{*} p\right)<3070 \mathrm{MeV}$ and $3130<M\left(D^{*} p\right)<3210 \mathrm{MeV}$, scaled by a factor of $3 / 16$ to account for the different widths of the sample regions. Away from the $D^{*}$ peak, the distribution in $\Delta M_{D^{*}}$ from the $M\left(D^{*} p\right)$ side bands gives a good description of that from the $M\left(D^{*} p\right)$ signal region, in both shape and normalisation. However, there is a clear difference around the expected value of $\Delta M_{D^{*}}$ for true $D^{*}$ mesons, where the distribution from the signal region lies well above that from the side bands. The signal region in $M\left(D^{*} p\right)$ is thus significantly richer in $D^{*}$ mesons than is the case elsewhere in the distribution.

The protons are clearly identified at low momentum, where the most probable $\mathrm{d} E / \mathrm{d} x$ for protons is well separated from those for other particle species. The analysis has been repeated with the proton momentum restricted to $p(p)<1.2 \mathrm{GeV}$, the measured $\mathrm{d} E / \mathrm{d} x$ required to be larger than that for a minimum ionising particle by a factor of at least 1.15 and the proton likelihood requirement modified to $L_{p}>0.5$. With this tighter proton selection, the requirements on $z\left(D^{*}\right)$ and $p_{\mathrm{T}}(K)+p_{\mathrm{T}}(\pi)$ are removed. In figure 4 , the $M\left(D^{*} p\right)$ distribution for this selection is compared with the predictions of the background model described in section 3.1. The enhancement in the region $M\left(D^{*} p\right) \simeq 3100 \mathrm{MeV}$ remains visible. The candidate proton tracks in the signal region $\left(3085<M\left(D^{*} p\right)<3115 \mathrm{MeV}\right)$ have an average $\left\langle L_{p}\right\rangle=0.92$.

Several further checks of the particle identification have been carried out using the data. No signal is obtained when the $D^{*}$ selection is modified such that the "wrong charge $D$ " candidates are taken (figure 2a) or when the $D^{*}$ candidates are replaced by $K \pi \pi_{s}$ combinations from a side band of the $\Delta M_{D^{*}}$ distribution. No clear peaks are observed when the proton candidates are assumed to have the kaon or pion mass. The signal is also not observed when the proton mass hypothesis is retained, but the $\mathrm{d} E / \mathrm{d} x$ requirement is modified to select pion or kaon candidates rather than proton candidates. 


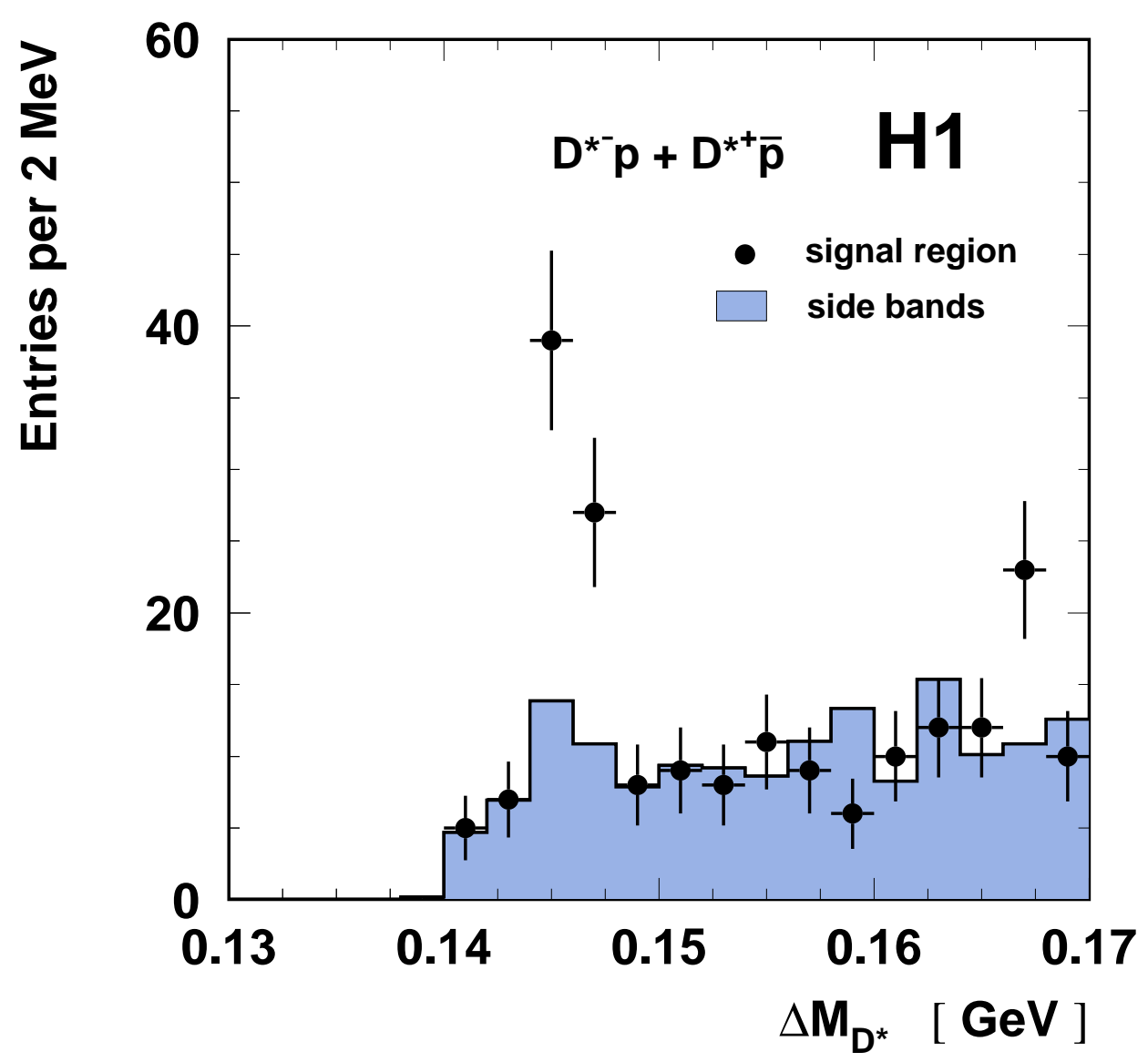

Figure 3: $\Delta M_{D^{*}}$ distribution for events in a $30 \mathrm{MeV}$ window about the signal in the oppositecharge $M\left(D^{*} p\right)$ distribution, with no requirement on $\Delta M_{D^{*}}$, compared with the corresponding distribution from side bands in the $M\left(D^{*} p\right)$ distribution, normalised according to the widths of the chosen sample regions.

\subsection{Kinematic and Reflection Tests}

Possible kinematic reflections that could fake the signal have been ruled out by studying invariant mass distributions and correlations involving the $K, \pi, \pi_{s}$ and proton candidates under various particle mass hypotheses. For example, there is no evidence for any resonant structure or correlations with $M\left(D^{*} p\right)$ in the invariant mass combinations $m(K p), m(\pi p)$ or $m\left(\pi_{s} p\right)$ of the proton candidates with the decay products of the $D^{*}$ meson.

Detailed studies have been carried out of the contribution to the $M\left(D^{*} p\right)$ distribution from the neutral, orbitally excited, P-wave $D_{1}^{0}(2420)$ and $D_{2}^{0 *}(2460)$ mesons and their charge conjugates [12], both of which decay to $D^{* \pm} \pi^{\mp}$. A simulation of the $D_{1}^{0} \rightarrow D^{*} \pi$ and $D_{2}^{0 *} \rightarrow D^{*} \pi$ decays with the PYTHIA [20] Monte Carlo generator is used to estimate their contribution to the observed signal. The simulated widths are set to the results from recent measurements [21] and the normalisation is obtained from the observed $D_{1}^{0}$ and $D_{2}^{0 *}$ yields in the data, as obtained from the distribution in $M\left(D^{*} \pi\right)=m\left(K^{\mp} \pi^{ \pm} \pi_{s}^{ \pm} \pi^{\mp}\right)-m\left(K^{\mp} \pi^{ \pm} \pi_{s}^{ \pm}\right)+m\left(D^{*}\right)$. The reflections due to the $D_{1}^{0}$ and $D_{2}^{0 *}$ mesons when the decay pion is misidentified as a proton yield a broad distribution in $M\left(D^{*} p\right)$, with a maximum below the signal region. The predicted contribution 


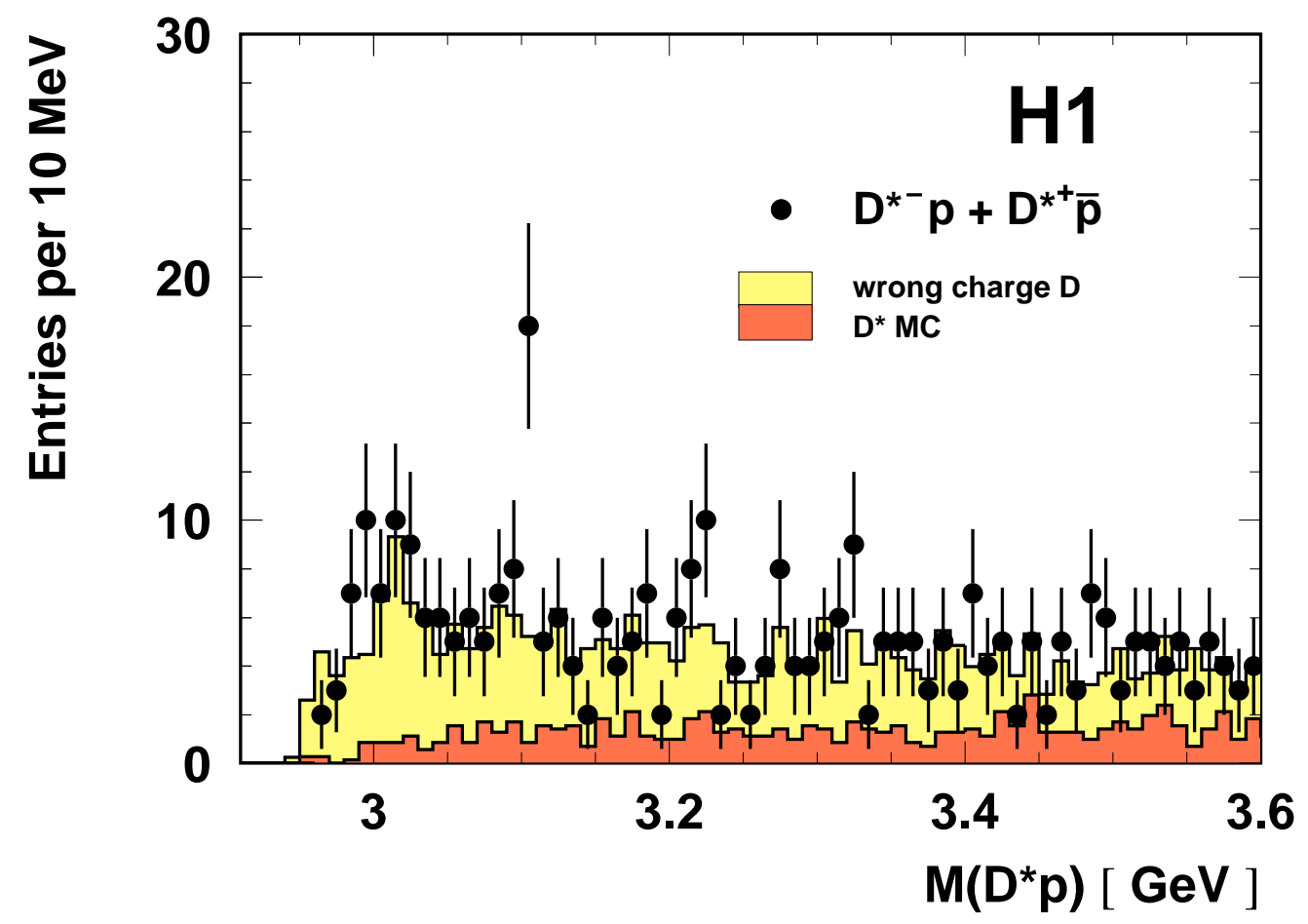

Figure 4: $M\left(D^{*} p\right)$ distribution for a low momentum proton selection with $p(p)<1.2 \mathrm{GeV}$. The data are compared with a two-component background model in which "wrong charge $D$ " $K^{ \pm} \pi^{ \pm}$combinations are used to describe non-charm related background and the " $D^{*}$ MC" simulation describes background involving real $D^{*}$ mesons.

within $\pm 24 \mathrm{MeV}$ of the observed peak is approximately four events. The signal in the measured $M\left(D^{*} p\right)$ distribution covers the full available phase space in $M\left(D^{*} \pi\right)$, with no evidence for enhancements in the region of the $D_{1}^{0}$ and $D_{2}^{0 *}$ mesons. The possibility of reflections involving the $D_{s 1}(2536)$ or $D_{s J}(2573)$, are similarly ruled out. ${ }^{5}$

The kinematics of the $D^{*}$ and proton candidates from the decay of a resonance would be expected to be different from those of the background distribution. Such a difference is observed for the opposite-charge $D^{*} p$ signal, as illustrated in figure 5a. The momentum distribution $p(p)$ is shown for all particles of opposite charge to the $D^{*}$ candidate which lead to entries in the signal and side band regions of $M\left(D^{*} p\right)$, as defined in section 3.2. No requirements are placed on the proton likelihood. The two side bands with larger and smaller $M\left(D^{*} p\right)$ than the signal give rise to compatible momentum spectra, both of which are significantly softer than that in the signal region. A similar difference is observed when the $M\left(D^{*} p\right)$ side band is replaced by combinations which lie in the signal region of $M\left(D^{*} p\right)$, but fall in a side band of the $\Delta M_{D^{*}}$ distribution.

Figure 5a suggests that, with no proton $\mathrm{d} E / \mathrm{d} x$ requirements, the signal-to-background ratio improves as $p(p)$ increases. In figure $5 \mathrm{~b}$, the $M\left(D^{*} p\right)$ distribution is shown for $p(p)>2 \mathrm{GeV}$,

\footnotetext{
${ }^{5}$ Given the proximity of the mass of the observed resonance to the $J / \psi$ mass, possible backgrounds involving $J / \psi$ decays have been considered. Baryon number and other conservation laws would be violated by the decay of the $J / \psi$ to $D^{*} p$. If the signal were due to $J / \psi$ decays with misidentified particles, the reconstructed mass would no longer lie at the $J / \psi$ value.
} 

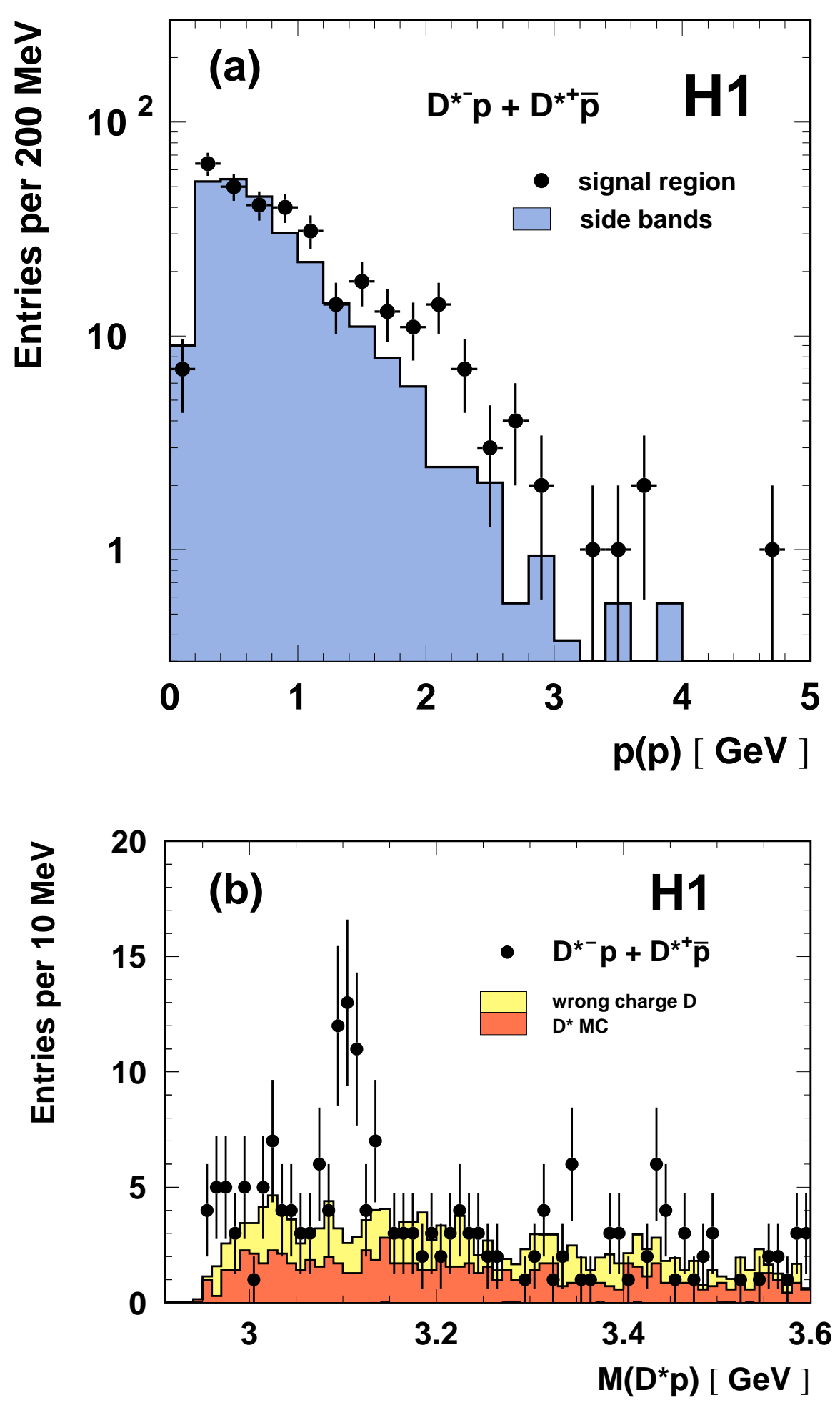

Figure 5: (a) Momentum distributions for all charged particles yielding $M\left(D^{*} p\right)$ values falling in the signal and side band regions of $M\left(D^{*} p\right)$, as defined in section 3.2, when combined with $D^{*}$ candidates of opposite charge. (b) $M\left(D^{*} p\right)$ distribution for $p(p)>2 \mathrm{GeV}$, with no proton $\mathrm{d} E / \mathrm{d} x$ requirements. The data are compared with a two-component background model in which "wrong charge $D$ " $K^{ \pm} \pi^{ \pm}$combinations are used to describe non-charm related background and the " $D^{*} \mathrm{MC}$ " simulation describes background involving real $D^{*}$ mesons. 
with no requirement on the proton likelihood. A strong signal is observed, with a reduced background which remains well described by the background model. The peak value and width of the observed signal are compatible with those for the standard selection shown in figure 2 a.

\subsection{Photoproduction Analysis}

The analysis has also been carried out using an independent sample provided by $\mathrm{H} 1$ data from the photoproduction region, where the scattered electron passes at a small angle into the backward beampipe, implying $Q^{2} \lesssim 1 \mathrm{GeV}^{2}$. The hadronic final state is used to reconstruct $y$ [22] and the selection $0.2<y<0.8$ is imposed. The combinatorial background to the $D^{*}$ selection is significantly larger for photoproduction than for DIS. To compensate for this, tighter proton and $D^{*}$ selections are imposed. The cut on the $D^{*}$ transverse momentum is modified to $p_{\mathrm{T}}\left(D^{*}\right)>2 \mathrm{GeV}$. The region $1.6<p(p)<2.0 \mathrm{GeV}$, around the point at which the $\mathrm{d} E / \mathrm{d} x$ parameterisations for protons and pions cross, is excluded and the requirement $L_{p}>0.25$ is made elsewhere.

The distribution in $M\left(D^{*} p\right)$ for opposite-charge $D^{*} p$ combinations in photoproduction is shown in figure 6. Again, a clear signal is observed near $M\left(D^{*} p\right)=3100 \mathrm{MeV}$, with mass and width compatible with those in the DIS case. The background distribution is reasonably modelled by the "wrong charge $D$ " selection. The photoproduction signal is also separately observed for $p(p)<1.6 \mathrm{GeV}$ and for $p(p)>2.0 \mathrm{GeV}$.

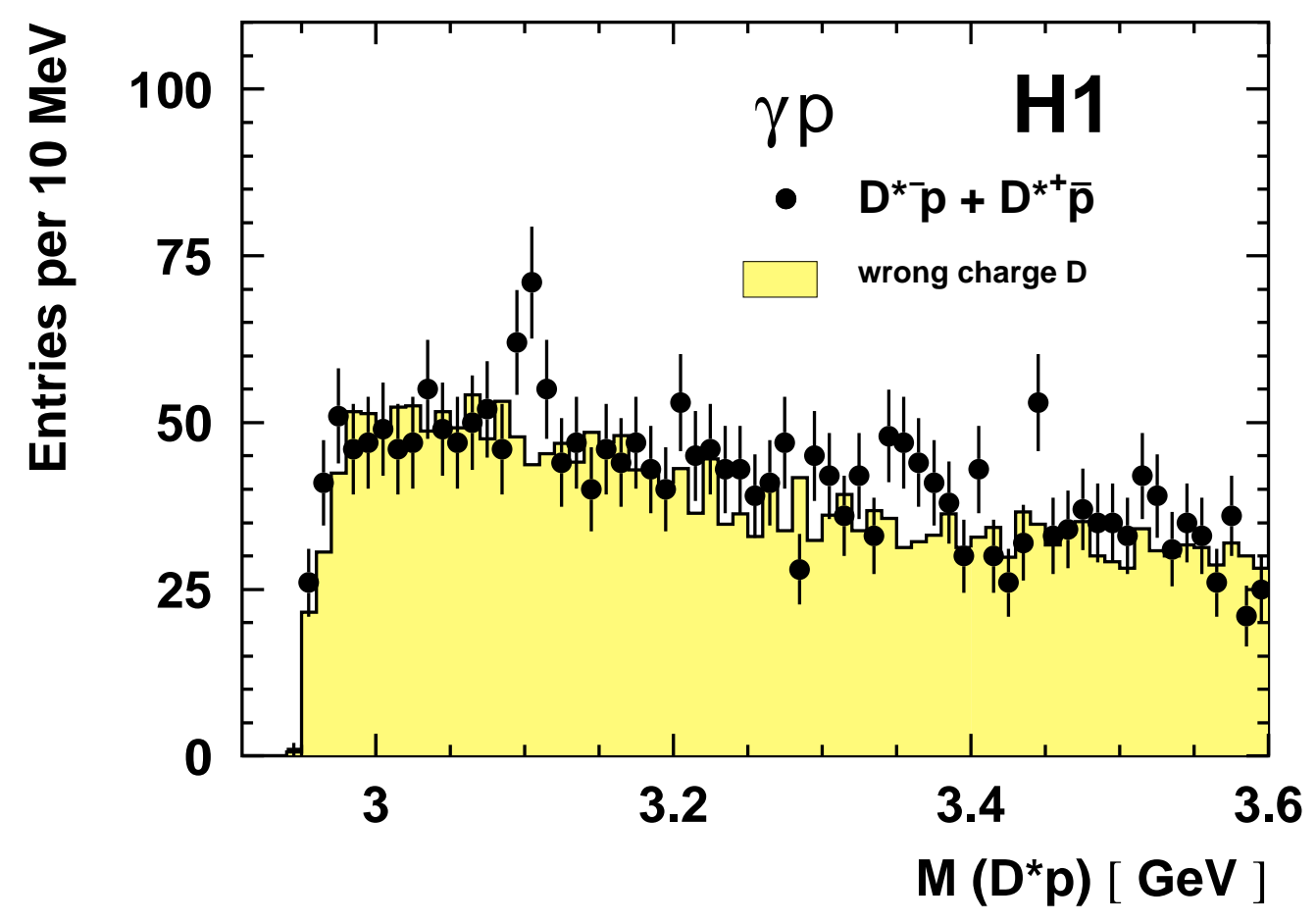

Figure 6: $M\left(D^{*} p\right)$ distribution from the photoproduction analysis, compared with a background model derived from "wrong charge $D^{\prime \prime} K^{ \pm} \pi^{ \pm}$combinations. 


\section{Signal Assessment}

Fits to the $M\left(D^{*} p\right)$ distribution from the opposite-charge $D^{*} p$ combinations in DIS have been carried out to evaluate the peak position, width and statistical significance. Assuming the measured width to be dominated by the experimental resolution, a Gaussian distribution is used for the signal, with the peak position, the width and the normalisation as free parameters. The background is parameterised with a power law of the form $\alpha\left[M\left(D^{*} p\right)-m\left(D^{*}\right)\right]^{\beta}$, with $\alpha$ and $\beta$ as free parameters. A log-likelihood fit is made in the range $2950<M\left(D^{*} p\right)<3600 \mathrm{MeV}$.

The results of this fit are compared with the data in figure 7. They are also summarised in table 2 , together with the results of separate fits to the $D^{*-} p$ and $D^{*+} \bar{p}$ contributions. The fit yields a peak position of $M\left(D^{*} p\right)=3099 \pm 3$ (stat.) MeV. The root-mean-square (RMS) width of the Gaussian is $12 \pm 3$ (stat.) $\mathrm{MeV}$, compatible with the experimental resolution of $7 \pm 2 \mathrm{MeV}$, as determined from a simple simulation of the observed resonance with zero width and an isotropic decay distribution. The signal consists of $N_{s}=50.6 \pm 11.2$ events, from which the observed $D^{*} p$ resonance is estimated to contribute roughly $1 \%$ of the total $D^{*}$ production rate in the kinematic region studied. The fit results are not significantly affected when the background parameterisation is replaced with a polynomial or when the full distribution is fitted with the inclusion of a function to describe the rise at threshold. The results are stable against shifts in the binning, changes to the bin width in the range $1 \mathrm{MeV}$ to $20 \mathrm{MeV}$ and variations in the selection criteria which do not significantly alter the signal-to-background ratios for the $D^{*}$ or proton candidates.

\begin{tabular}{|l|c|c|c|}
\hline Sample & $\begin{array}{c}\text { Mass } \\
(\mathbf{M e V})\end{array}$ & $\begin{array}{c}\text { Gaussian width } \\
(\mathbf{M e V})\end{array}$ & $\boldsymbol{N}_{\boldsymbol{s}}$ \\
\hline $\boldsymbol{D}^{*+} \overline{\boldsymbol{p}}+\boldsymbol{D}^{*-} \boldsymbol{p}$ & $3099 \pm 3$ & $12 \pm 3$ & $50.6 \pm 11.2$ \\
$\boldsymbol{D}^{*-} \boldsymbol{p}$ & $3102 \pm 3$ & $9 \pm 3$ & $25.8 \pm 7.1$ \\
$\boldsymbol{D}^{*+} \overline{\boldsymbol{p}}$ & $3096 \pm 6$ & $13 \pm 6$ & $23.4 \pm 8.6$ \\
\hline
\end{tabular}

Table 2: Results of the fit as described in the text for opposite-charge $D^{*} p$ combinations. The fitted position and Gaussian RMS width of the peak are given, together with the total number of signal events $\left(N_{s}\right)$. The statistical uncertainties quoted take account of the correlations between the variable parameters in the fit.

The systematic uncertainty on the mass of the peak is $5 \mathrm{MeV}$, estimated from the reconstructed masses of known states, such as the $J / \psi$, with decays to particles in a similar momentum range, and from the variations in the peak position when the fitting procedure or selection criteria are modified.

The probability that the background distribution fluctuates to produce the signal has been evaluated by comparing the observed number of events with background estimates for a window spanning $3075<M\left(D^{*} p\right)<3123 \mathrm{MeV}$, corresponding to $\pm 2 \sigma$ about the peak position according to the fit. The total number of events in this interval is 95 . The background contribution estimated using the fit described above is $N_{b}=45.0 \pm 2.8$ (stat.). A parameterisation of the background model shown in figure 2a yields a consistent value for $N_{b}$. A more conservative approach is to fit only the power-law background function to the full $M\left(D^{*} p\right)$ distribution. 


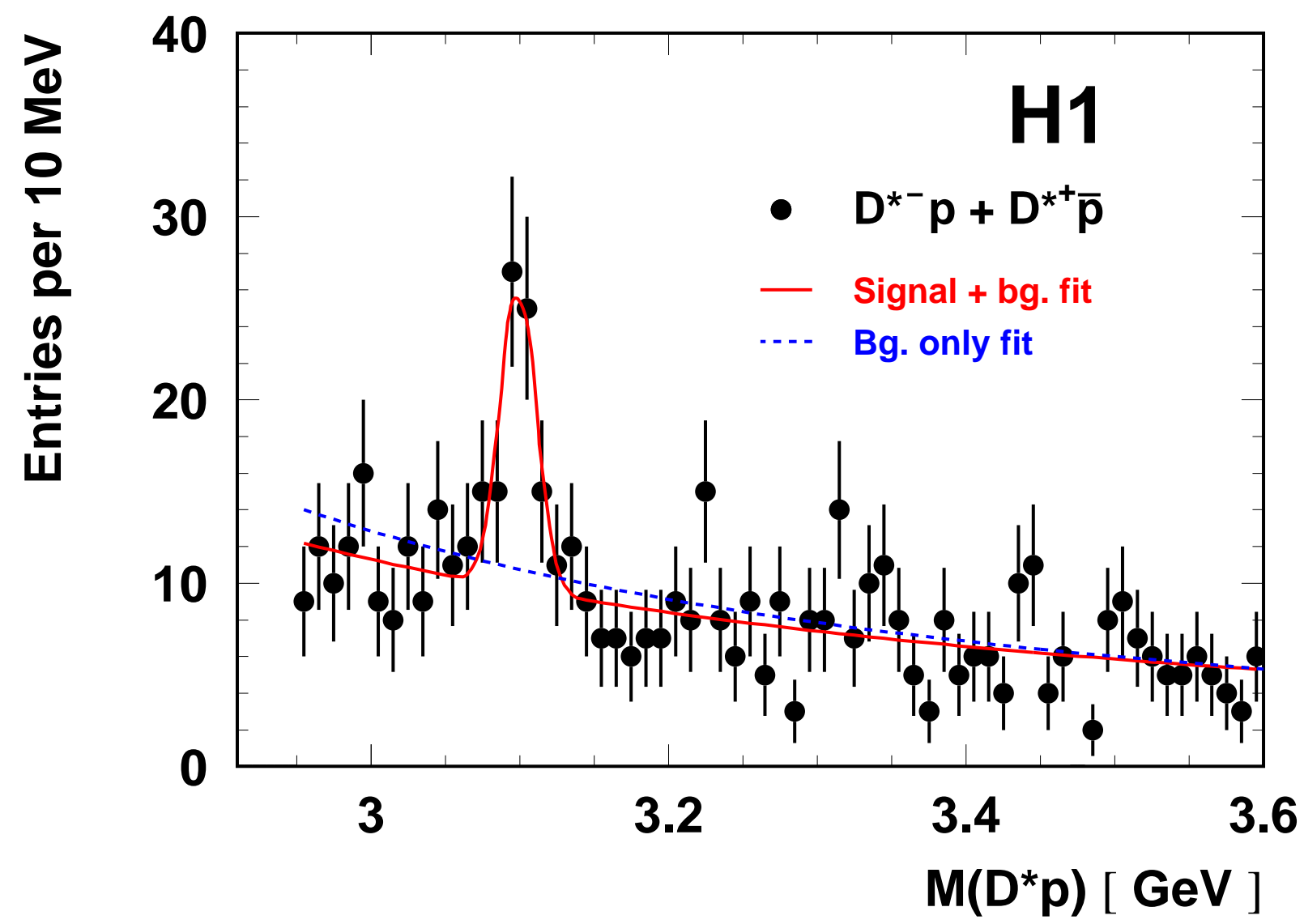

Figure 7: $M\left(D^{*} p\right)$ distribution from opposite-charge $D^{*} p$ combinations in DIS, compared with the results of a fit in which both signal and background components are included (solid line) and with the results of a fit in which only the background component is included (dashed line).

The data are compared with the result of such a fit in figure 7 . The corresponding background estimate is $N_{b}=51.7 \pm 2.7$ (stat.). The probability that a background of $N_{b}=51.7$ events fluctuates to produce at least the number of events in the signal is $4 \cdot 10^{-8}$, assuming Poisson statistics. This probability corresponds to $5.4 \sigma$ when expressed as an equivalent number of Gaussian standard deviations. From the change in maximum $\log$-likelihood $\Delta(\ln \mathcal{L})$ when the full distribution is fitted under the null and signal hypotheses, corresponding to the two curves shown in figure 7, the statistical significance is estimated to be $\sqrt{-2 \Delta(\ln \mathcal{L})}=6.2 \sigma$.

A state decaying strongly to $D^{*-} p$ must have baryon number +1 and charm -1 and thus has a minimal constituent quark composition of $u u d d \bar{c}$. The observed resonance is therefore a candidate for the charmed analogue $\theta_{c}^{0}[5,8]$ of the $\theta^{+}$. The narrow width is reminiscent of that in the strange case. Given the relatively large mass of the resonance, it is also a candidate for an excited state such as the $\theta_{c}^{* 0}$ with spin $3 / 2$ [23]. 


\section{Summary}

An investigation has been carried out of the invariant mass combinations of $D^{*}$ and proton candidates using $\mathrm{H} 1$ deep inelastic electron-proton scattering data. A clear and narrow resonance is observed for both $D^{*-} p$ and $D^{*+} \bar{p}$ combinations with an invariant mass of $M\left(D^{*} p\right)=$ $3099 \pm 3$ (stat.) \pm 5 (syst.) $\mathrm{MeV}$. The probability for the background distribution to fluctuate to produce a signal as large as that observed is less than $4 \cdot 10^{-8}$. The region of $M\left(D^{*} p\right)$ in which the signal is observed contains a richer yield of $D^{*}$ mesons and exhibits a harder proton candidate momentum distribution than is the case for side bands in $M\left(D^{*} p\right)$. The measured RMS width of the resonance is $12 \pm 3$ (stat.) $\mathrm{MeV}$, consistent with the experimental resolution. A signal with compatible mass and width is also observed in an independent photoproduction data sample.

The resonance is interpreted as an anti-charmed baryon decaying to $D^{*-} p$ and its charge conjugate decaying to $D^{*+} \bar{p}$. The minimal constituent quark composition of such a baryon is $u u d d \bar{c}$, making it a candidate for a charmed pentaquark state.

\section{Acknowledgments}

We are grateful to the HERA machine group whose outstanding efforts have made this experiment possible. We thank the engineers and technicians for their work in constructing and now maintaining the $\mathrm{H} 1$ detector, our funding agencies for financial support, the DESY technical staff for continual assistance and the DESY directorate for support and for the hospitality which they extend to the non-DESY members of the collaboration. 


\section{References}

[1] T. Nakano et al. [LEPS Collaboration], Phys. Rev. Lett. 91 (2003) 012002 [hepex/0301020];

V. V. Barmin et al. [DIANA Collaboration], Phys. Atom. Nucl. 66 (2003) 1715 [Yad. Fiz. 66 (2003) 1763] [hep-ex/0304040];

S. Stepanyan et al. [CLAS Collaboration], Phys. Rev. Lett. 91 (2003) 252001 [hepex/0307018];

J. Barth et al. [SAPHIR Collaboration], Phys. Lett. B572 (2003) 127 [hep-ex/0307083];

A. E. Asratyan, A. G. Dolgolenko and M. A. Kubantsev, submitted to Phys. Atom. Nucl. [hep-ex/0309042];

V. Kubarovsky et al. [CLAS Collaboration], Phys. Rev. Lett. 92 (2004) 032001; erratumibid. 92 (2004) 049902 [hep-ex/0311046];

A. Airapetian et al. [HERMES Collaboration], submitted to Phys. Lett. B [hepex/0312044];

A. Aleev et al. [SVD Collaboration], submitted to Yad. Fiz. [hep-ex/0401024];

M. Abdel-Bary et al. [COSY-TOF Collaboration], [hep-ex/0403011].

[2] R. Jaffe, SLAC-PUB-1774, talk presented at the Topical Conf. on Baryon Resonances, Oxford, UK, 1976;

H. Høgaasen and P. Sorba, Nucl. Phys. B 145 (1978) 119;

D. Strottman, Phys. Rev. D20 (1979) 748;

C. Roisnel, Phys. Rev. D20 (1979) 1646.

[3] D. Diakonov, V. Petrov and M. V. Polyakov, Z. Phys. A359 (1997) 305 [hep-ph/9703373].

[4] C. Alt et al. [NA49 Collaboration], Phys. Rev. Lett. 92 (2004) 042003 [hep-ex/0310014].

[5] R. L. Jaffe and F. Wilczek, Phys. Rev. Lett. 91 (2003) 232003 [hep-ph/0307341].

[6] H. Walliser and V. B. Kopeliovich, J. Exp. Theor. Phys. 97 (2003) 433 [Zh. Eksp. Teor. Fiz. 124 (2003) 483] [hep-ph/0304058];

S. Capstick, P. R. Page and W. Roberts, Phys. Lett. B570 (2003) 185 [hep-ph/0307019];

M. Karliner and H. Lipkin, Phys. Lett. B575 (2003) 249 [hep-ph/0307243];

D. E. Kahana and S. H. Kahana, [hep-ph/0310026];

E. Shuryak and I. Zahed, [hep-ph/0310270].

[7] H. Lipkin, Phys. Lett. B195 (1987) 484;

C. Cignoux, B. Silvestre-Brac and J. Richard, Phys. Lett. B193 (1987) 323;

D. Riska, N. Scoccola, Phys. Lett. B299 (1993) 338;

F. Stancu, Phys. Rev. D58 (1998) 111501 [hep-ph/9803442].

[8] M. Karliner and H. J. Lipkin, [hep-ph/0307343];

K. Cheung, [hep-ph/0308176].

[9] J. Steinhart, 'Die Messung des totalen $c \bar{c}$-Photoproduktions-Wirkungsquerschnittes durch die Rekonstruktion von $\Lambda_{c}$ Baryonen unter Verwendung der verbesserten $\mathrm{d} E / \mathrm{d} x$ Teilchenidentifikation am H1 Experiment bei HERA', Ph.D. thesis, 1999, Universität Hamburg (in 
German, available from

http://www-hl.desy.de/publications/theses_list.html).

[10] H1 Collaboration, I. Abt et al., Nucl. Inst. Meth. A386 (1997) 310;

H1 Collaboration, I. Abt et al., Nucl. Inst. Meth. A386 (1997) 348.

[11] S. Aid et al. [H1 Collaboration], Nucl. Phys. B472 (1996) 32 [hep-ex/9604005];

C. Adloff et al. [H1 Collaboration], Nucl. Phys. B545 (1999) 21 [hep-ex/9812023];

C. Adloff et al. [H1 Collaboration], Phys. Lett. B528 (2002) 199 [hep-ex/0108039].

[12] Particle Data Group, H. Hagiwara et al., Phys. Rev. D66 (2002) 010001.

[13] G. Feldman et al., Phys. Rev. Lett. 38 (1977) 1313.

[14] H. Jung, Comp. Phys. Commun. 86 (1995) 147;

H. Jung, 'The RAPGAP Monte Carlo for Deep Inelastic Scattering, version 2.08', Lund University, 1999, (http://www.desy.de/ jung/rapgap.html).

[15] B. Andersson, G. Gustafson, G. Ingelman and T. Sjöstrand, Phys. Rept. 97 (1983) 31.

[16] T. Sjöstrand, Comp. Phys. Commun. 82 (1994) 74 and LU-TP-95-20 [hep-ph/9508391].

[17] A. Kwiatkowski, H. Spiesberger and H.J. Möhring, Comp. Phys. Commun. 69 (1992) 155;

L. Lönnblad, Comp. Phys. Commun 71 (1992) 15;

K. Charchula, G.A. Schuler and H. Spiesberger, Comp. Phys. Commun 81 (1994) 381, (http://www.desy.de/ ^hspiesb/djangoh.html).

[18] H. Jung and G. P. Salam, Eur. Phys. J. C19 (2001) 351 [hep-ph/0012143];

H. Jung, Comput. Phys. Commun. 143 (2002) 100 [hep-ph/0109102].

[19] G. Marchesini et al., Comp. Phys. Commun. 67 (1992) 465.

[20] T. Sjöstrand et al., Comp. Phys. Commun. 135 (2001) 238 [hep-ph/0010017].

[21] K. Abe et al. [Belle Collaboration], submitted to Phys. Rev. D [hep-ex/0307021].

[22] A. Blondel and F. Jacquet, Proceedings of the Study for an $e p$ Facility for Europe, ed. U. Amaldi, DESY 79-48 (1979) 391.

[23] J. J. Dudek and F. E. Close, Phys. Lett. B583 (2004) 278 [hep-ph/0311258];

B. Wu and B. Ma, [hep-ph/0402244]. 